\title{
The Asymptotic Behavior for a Class of Impulsive Delay Differential Equations
}

\author{
Zhichun Yang \\ Department of Mathematics, Chongqing Normal University, Chongqing 400047, China \\ Correspondence should be addressed to Zhichun Yang; zhichy@yahoo.com.cn
}

Received 27 January 2013; Accepted 21 March 2013

Academic Editor: Chuangxia Huang

Copyright (C) 2013 Zhichun Yang. This is an open access article distributed under the Creative Commons Attribution License, which permits unrestricted use, distribution, and reproduction in any medium, provided the original work is properly cited.

\begin{abstract}
This paper is concerned with asymptotical behavior for a class of impulsive delay differential equations. The new criteria for determining attracting sets and attracting basin of the impulsive system are obtained by developing the properties of quasi-invariant sets. Examples and numerical simulations are given to illustrate the effectiveness of our results. In addition, we show that the impulsive effects may play a key role to these asymptotical properties even though the solutions of corresponding nonimpulsive systems are unbounded.
\end{abstract}

\section{Introduction}

Impulsive delay differential equations have attracted increasing interests since time delays and impulsive effects commonly exist in many fields such as population dynamics, automatic control, drug administration, and communication networks [1-4]. In past two decades, its asymptotical behaviors such as stability and attractivity of the equilibrium point or periodical solutions have been deeply studied for impulsive functional differential equations (see, [5-18]).

However, under impulsive perturbation, the solutions may not be attracted to an equilibrium point or periodical trajectory but to some bounded region. In this case, it is interesting to investigate the attracting set and attracting basin, that is, the region attracting the solutions and the range in which initial values vary when remaining the attractivity for impulsive delay differential equations. In [19], Xu and Yang first give the method to estimate global attracting set and invariant set for impulsive delayed systems by developing delayed differential inequalities. The techniques are further developed to study global attractivity for some complex impulsive systems such as impulsive neutral differential equations [20,21] and impulsive stochastic systems [22]. But the techniques and methods given in the existing publications are invalid for determining locally attracting set and attracting basin for impulsive delay differential equations.
In this paper, our objective is to mainly discuss the asymptotical behavior on (locally) attracting set and its attracting basin for a class of impulsive delay differential equations. Based on the quasi-invariant properties, we estimate the existence range of attracting set and attracting basin of the impulsive delay systems by solving algebraic equations and employing differential inequality technique. Examples are given to illustrate the effectiveness of our method and show that the asymptotic behavior of the impulsive systems may be different from one of the corresponding continuous systems.

\section{Preliminaries}

Let $N$ be the set of all positive integers, $R^{n}$ the space of $n$ dimensional real column vectors, and $R^{m \times n}$ the set of $m \times n$ real matrices. For $A, B \in R^{m \times n}$ or $A, B \in R^{n}, A \geq B(A \leq$ $B, A>B, A<B$ ) means that each pair of corresponding elements of $A$ and $B$ satisfies the inequality " $\geq(\leq,>,<)$." $R_{+}^{n}=$ $\left\{x \in R^{n} \mid x \geq 0\right\}, E=(1,1, \ldots, 1)^{T} \in R^{n}$, and $I$ denotes an $n \times n$ unit matrix.

Let $\tau>0$ and $t_{0}<t_{1}<t_{2}<\cdots$ be the fixed points with $\lim _{k \rightarrow \infty} t_{k}=\infty$ (called impulsive moments).

$C[X, Y]$ denotes the space of continuous mappings from the topological space $X$ to the topological space $Y$. Let $C \stackrel{\Delta}{=}$ $C\left[[-\tau, 0], R^{n}\right]$ especially. 
Morever, PC $\triangleq$ 至 $\left\{\phi:[-\tau, 0] \rightarrow R^{n} \mid \phi\left(t^{+}\right)=\phi(t)\right.$ for $t \in[-\tau, 0), \phi\left(t^{-}\right)$exists for $t \in(-\tau, 0], \phi\left(t^{-}\right)=\phi(t)$ for all but at most a finite number of points $t \in(-\tau, 0]\}$. PC is a space of piecewise right-hand continuous functions which is a nature extension of the phrase space $C$.

We define PC $\left[\left[t_{0}, \infty\right), R^{n}\right] \stackrel{\Delta}{=}\left\{\psi:\left[t_{0}, \infty\right) \rightarrow R^{n} \mid \psi(t)\right.$ is continuous at $t \neq t_{k}, \psi\left(t_{k}^{+}\right)$and $\psi\left(t_{k}^{-}\right)$exist, $\psi\left(t_{k}\right)=\psi\left(t_{k}^{+}\right)$, for $k \in N\}$.

For $x \in R^{n}, A \in R^{n \times n}, \phi \in C$ or $\phi \in \mathrm{PC}$, we define

$$
\begin{gathered}
{[x]^{+}=\left(\left|x_{1}\right|,\left|x_{2}\right|, \ldots,\left|x_{n}\right|\right)^{T},} \\
{[A]^{+}=\left(\left|a_{i j}\right|\right)_{n \times n},} \\
{[\phi]_{\tau}^{+}=\left(\left\|\phi_{1}\right\|_{\tau},\left\|\phi_{2}\right\|_{\tau}, \ldots,\left\|\phi_{n}\right\|_{\tau}\right),}
\end{gathered}
$$

where $\left\|\phi_{i}\right\|_{\tau}=\sup _{s \in[-\tau, 0]}\left\|\phi_{i}(s)\right\|$ and $\|\cdot\|$ is an norm in $R^{n}$.

In this paper, we will consider a impulsive delay differential equations:

$$
\begin{gathered}
\dot{x}(t)=A x(t)+f\left(t, x_{t}\right), \quad t \neq t_{k}, t \geq t_{0}, \\
\Delta x=B x\left(t_{k}^{-}\right)+I_{k}\left(t_{k}^{-}, x\left(t_{k}^{-}\right)\right), \quad k \in N,
\end{gathered}
$$

where $\dot{x}(t)$ denotes the right-hand derivative of $x(t), \Delta x=$ $x\left(t_{k}^{+}\right)-x\left(t_{k}^{-}\right), x\left(t_{k}\right)=x\left(t_{k}^{+}\right), A=\operatorname{diag}\left\{a_{1}, a_{2}, \ldots, a_{n}\right\}, B=$ $\operatorname{diag}\left\{b_{1}, b_{2}, \ldots, b_{n}\right\}, f \in C\left[\left[t_{k-1}, t_{k}\right) \times \mathrm{PC}, R^{n}\right]$, and the limit $\lim _{(t, \phi) \rightarrow\left(t_{k}^{-}, \varphi\right)} f(t, \phi)=f\left(t_{k}^{-}, \varphi\right)$ exists, $I_{k} \in C\left[\left[t_{0}, \infty\right) \times\right.$ $\left.R^{n}, R^{n}\right]$, and $x_{t} \in \mathrm{PC}$ is defined by $x_{t}(s)=x(t+s), s \in[-\tau, 0]$.

A function $x(t):\left[t_{0}-\tau, \infty\right) \rightarrow R^{n}$ is called to be a solution of (2) through $\left(t_{0}, \phi\right)$, if $x(t) \in \mathrm{PC}\left[\left[t_{0}, \infty\right), R^{n}\right]$ as $t \geq t_{0}$, and satisfies (2) with the initial condition

$$
x\left(t_{0}+s\right)=\phi(s), \quad s \in[-\tau, 0], \phi \in \mathrm{PC} .
$$

Throughout the paper, we always assume that for any $\phi \in \mathrm{PC}$, system (2) has at least one solution through $\left(t_{0}, \phi\right)$, denoted by $x\left(t, t_{0}, \phi\right)$ or $x_{t}\left(t_{0}, \phi\right)$ (simply $x(t)$ and $x_{t}$ if no confusion should occur), where $x_{t}\left(t_{0}, \phi\right)=x\left(t+s, t_{0}, \phi\right) \in \mathrm{PC}, s \in$ $[-\tau, 0]$.

In this paper, we need the following definitions involving attracting set, attracting basin, the quasi-invariant set of impulsive systems, and monotonous vector functions.

Definition 1. The set $S \subset \mathrm{PC}$ is called to be an attracting set of (2), and $D \subset P C$ is called an attraction basin of $S$, if for any initial value $\phi \in D$, the solution $x_{t}\left(t_{0}, \phi\right)$ converges to $S$ as $t \rightarrow+\infty$. That is,

$$
\operatorname{dist}\left(x_{t}\left(t_{0}, \phi\right), S\right) \longrightarrow 0, \quad \text { as } t \longrightarrow+\infty,
$$

where $\operatorname{dist}(\varphi, S)=\inf _{\psi \in S} \operatorname{dist}(\varphi, \psi), \operatorname{dist}(\varphi, \psi)=\sup _{s \in[-\tau, 0]}$ $\|\varphi(s)-\psi(s)\|$, for $\varphi \in$ PC.

Definition 2. The set $D \subset \mathrm{PC}$ is called to be a positive quasiinvariant set of (2), if there is a positive diagonal matrix $L=\operatorname{diag}\left\{l_{i}\right\}$ such that for any initial value $\phi \in D$, the solutions $x_{t}\left(t_{0}, \phi\right)$ satisfy $L x_{t}\left(t_{0}, \phi\right) \in D$, for $t \geq t_{0}$. When $L=$ $I$ (identity matrix) especially, the set $D$ is called positively invariant.
Definition 3. Let $\Omega \subset R^{n}$. The vector function $F(x): \Omega \rightarrow$ $R^{n}$ is called to be monotonically nondecreasing in $\Omega$, if for any $x^{\prime}, x^{\prime \prime} \in \Omega, x^{\prime} \leq x^{\prime \prime}$ implies $F\left(x^{\prime}\right) \leq F\left(x^{\prime \prime}\right)$.

\section{Main Results}

In this paper, we always make the following assumptions.

$\left(H_{1}\right)$ There exist nonnegative constants $\theta, \varrho$ such that $0<$ $\theta \leq t_{k}-t_{k-1} \leq \varrho$, for $k \in N$.

$\left(H_{2}\right)[f(t, \varphi)]^{+} \leq p\left([\varphi]_{\tau}^{+}\right)$for $t \geq t_{0}$ and $\varphi \in$ PC, where the vector function $p(\cdot): R_{+}^{n} \rightarrow R_{+}^{n}$ is continuous and monotonically nondecreasing in $R_{+}^{n}$.

$\left(H_{3}\right)\left[I_{k}(t, x)\right]^{+} \leq q\left([x]^{+}\right)$, for $t \geq t_{0}, k \in N$ and $x \in$ $R^{n}$, where the vector function $q(\cdot): R_{+}^{n} \rightarrow R_{+}^{n}$ is continuous and monotonically nondecreasing in $R_{+}^{n}$.

To obtain attractivity, we first give the quasi-invariant properties of (2).

Theorem 4. Assume that in addition to $\left(H_{1}\right)-\left(H_{3}\right)$, there is a vector $z^{*} \geq 0$ such that

$$
p\left(M z^{*}\right)+W\left[I-e^{-W \theta}\right]^{-1} q\left(M z^{*}\right)-W z^{*}<0,
$$

where $W=\operatorname{diag}\left\{w_{1}, \ldots, w_{n}\right\}, M=\operatorname{diag}\left\{m_{1}, \ldots, m_{n}\right\}, w_{i}>$ $0, m_{i} \geq 1, i=1,2, \ldots, n$, are defined by

$$
\begin{aligned}
& w_{i}= \begin{cases}-a_{i}-\frac{\ln \left|1+b_{i}\right|}{\varrho}, & \text { if } 0<\left|1+b_{i}\right|<1, \\
-a_{i}-\frac{\ln \left|1+b_{i}\right|}{\theta}, & \text { if }\left|1+b_{i}\right| \geq 1,\end{cases} \\
& m_{i}= \begin{cases}\frac{1}{\left|1+b_{i}\right|}, & \text { if } 0<\left|1+b_{i}\right|<1, \\
1, & \text { if }\left|1+b_{i}\right| \geq 1 .\end{cases}
\end{aligned}
$$

Then, the set $D=\left\{\phi \in P C \mid[\phi]_{\tau}^{+} \leq z^{*}\right\}$ is a positive quasiinvariant set of (2). When $M=I$ especially, $D$ is a positive invariant set of (2).

Proof. Let $x(t)=x\left(t, t_{0}, \phi\right)$ be a solution of (2) through $\left(t_{0}, \phi\right)$. It is easily verified that the following formula for the variation of parameters is valid:

$$
\begin{aligned}
x(t)= & K\left(t, t_{0}\right) \phi(0)+\int_{t_{0}}^{t} K(t, s) f\left(s, x_{s}\right) d s \\
& +\sum_{t_{0}<t_{k} \leq t} K\left(t, t_{k}\right) I_{k}\left(t_{k}^{-}, x\left(t_{k}^{-}\right)\right), \quad t \geq t_{0},
\end{aligned}
$$

where $K(t, s)$ is the Cauchy matrix of linear impulsive system

$$
\begin{cases}\dot{y}(t)=A y(t), & t \neq t_{k}, \\ \Delta y\left(t_{k}^{+}\right)=B y\left(t_{k}^{-}\right), & k \in N .\end{cases}
$$

According to the representation of the Cauchy matrix (see page $74[2])$,

$$
K(t, s)=e^{A(t-s)} \prod_{s<t_{k} \leq t}(I+B), \quad t \geq s \geq t_{0} .
$$


Since $0<\theta \leq t_{k}-t_{k-1} \leq \varrho$, for $k \in N$, we obtain the following estimate:

$$
\begin{aligned}
& \prod_{s<t_{k} \leq t}\left|1+b_{i}\right| \\
& \quad \leq \begin{cases}\left|1+b_{i}\right|^{((t-s) / \varrho)-1}=\frac{1}{\left|1+b_{i}\right|} e^{\left(\ln \left|1+b_{i}\right| / \varrho\right)(t-s)}, & \text { if } 0<\left|1+b_{i}\right|<1, \\
\left|1+b_{i}\right|^{(t-s) / \theta}=e^{\left(\ln \left|1+b_{i}\right| / \theta\right)(t-s)}, & \text { if }\left|1+b_{i}\right| \geq 1 .\end{cases}
\end{aligned}
$$

In terms of the definition of $M$ and $W$,

$$
[K(t, s)]^{+} \leq M e^{-W(t-s)}, \quad t \geq s \geq t_{0} .
$$

By (7) and (11) and the assumptions $\left(\mathrm{H}_{2}\right)$ and $\left(\mathrm{H}_{3}\right)$, then

$$
\begin{aligned}
{[x(t)]^{+} \leq } & M e^{-W\left(t-t_{0}\right)}[\phi]_{\tau}^{+}+M \int_{t_{0}}^{t} e^{-W(t-s)} p\left(\left[x_{s}\right]_{\tau}^{+}\right) d s \\
& +M \sum_{t_{0}<t_{k} \leq t} e^{-W\left(t-t_{k}\right)} q\left(\left[x\left(t_{k}^{-}\right)\right]^{+}\right), \quad t \geq t_{0} .
\end{aligned}
$$

Since $t_{k}-t_{k-1} \geq \theta>0$ and $W=\operatorname{diag}\left\{w_{1}, \ldots, w_{n}\right\}>0$, we have

$$
\begin{aligned}
\sum_{t_{0}<t_{k} \leq t} e^{-W\left(t-t_{k}\right)} & =\sum_{t_{0}<t_{k} \leq t} e^{-W\left(t-t_{k}\right)}\left[I-e^{-W \theta}\right]\left[I-e^{-W \theta}\right]^{-1} \\
& \leq \sum_{t_{0}<t_{k} \leq t} e^{-W\left(t-t_{k}\right)}\left[I-e^{-W\left(t_{k}-t_{k-1}\right)}\right]\left[I-e^{-W \theta}\right]^{-1} \\
& =\sum_{t_{0}<t_{k} \leq t}\left[e^{-W\left(t-t_{k}\right)}-e^{-W\left(t-t_{k-1}\right)}\right]\left[I-e^{-W \theta}\right]^{-1} \\
& \leq\left[I-e^{-W\left(t-t_{0}\right)}\right]\left[I-e^{-W \theta}\right]^{-1} .
\end{aligned}
$$

From the strict inequality (5), there is an enough small number $\varepsilon>0$ such that

$$
\begin{gathered}
p(M z)+W\left[I-e^{-W \theta}\right]^{-1} q(M z)-W z<0, \\
z \stackrel{\Delta}{=} z^{*}+\varepsilon E>0 .
\end{gathered}
$$

In the following, we will prove that $[\phi]_{\tau}^{+}<z$ implies

$$
[x(t)]^{+}=\left[x\left(t, t_{0}, \phi\right)\right]^{+}<M z, \quad t \geq t_{0} .
$$

Otherwise, from the piecewise continuity of $x(t)$, there must be an integer $i$ and $t^{*}>t_{0}$ such that

$$
\begin{gathered}
\left|x_{i}\left(t^{*}\right)\right| \geq m_{i} z_{i}, \\
{[x(t)]^{+} \leq M z, \quad t_{0} \leq t<t^{*} .}
\end{gathered}
$$

By using (12), (13), (14), (17), $W>0$, and the monotonicity of $p(\cdot), q(\cdot)$, we can get

$$
\begin{aligned}
{\left[x\left(t^{*}\right)\right]^{+} \leq } & e^{-W\left(t^{*}-t_{0}\right)} M[\phi]_{\tau}^{+}+M \int_{t_{0}}^{t^{*}} e^{-W\left(t^{*}-s\right)} p(M z) d s \\
& +M \sum_{t_{0}<t_{k} \leq t^{*}} e^{-W\left(t^{*}-t_{k}\right)} q(M z) \\
< & e^{-W\left(t^{*}-t_{0}\right)} M z+M\left(I-e^{-W\left(t^{*}-t_{0}\right)}\right) W^{-1} p(M z) \\
& +M\left(I-e^{-W\left(t^{*}-t_{0}\right)}\right)\left[I-e^{-W \theta}\right]^{-1} q(M z) \\
= & e^{-W\left(t^{*}-t_{0}\right)} M W^{-1} \\
& \times\left[W z-p(M z)-W\left[I-e^{-W \theta}\right]^{-1} q(M z)\right] \\
& +W{ }^{-1} M p(M z)+M\left[I-e^{-W \theta}\right]^{-1} q(M z) \\
< & M W^{-1}\left[W z-p(M z)-W\left[I-e^{-W \theta}\right]^{-1} q(M z)\right] \\
& +W{ }^{-1} M p(M z)+M\left[I-e^{-W \theta}\right]^{-1} q(M z) \\
= & M z .
\end{aligned}
$$

This contradicts (16), and so (15) holds. Letting $\varepsilon \rightarrow 0$, from (15), we have for any $\phi \in D$ (i.e., $[\phi]_{\tau}^{+} \leq z^{*}$ ),

$$
\begin{array}{r}
{\left[x\left(t, t_{0}, \phi\right)\right]^{+} \leq M z^{*}, \quad \text { that is, }\left[M^{-1} x\left(t, t_{0}, \phi\right)\right]^{+} \leq z^{*},} \\
t \geq t_{0} .
\end{array}
$$

Therefore, the set $D=\left\{\phi \in \mathrm{PC} \mid[\phi]_{\tau}^{+} \leq z^{*}\right\}$ is a positive quasi-invariant set of (2). When $M=I$ especially, $D$ is a positive invariant set of (2). The proof is complete.

Based on the obtained quasi-invariant set, we have the following

Theorem 5. Let

$$
\Delta(z)=p(z)+W\left[I-e^{-W \theta}\right]^{-1} q(z)-M^{-1} W z, \quad z \in R_{+}^{n} .
$$

Assume that all conditions in Theorem 4 hold. Define

$$
\begin{gathered}
\Omega_{1}=\left\{z \in R_{+}^{n} \mid \Delta(M z)<0\right\}, \\
\Omega_{2}=\left\{z \in R_{+}^{n} \mid \Delta(z)<0\right\}, \\
\Omega_{3}=\left\{z \in R_{+}^{n} \mid \Delta(z) \geq 0\right\}, \\
\Omega_{1}^{*}=\bigcup_{z^{*} \in \Omega_{1}}\left\{z \in R_{+}^{n} \mid z \leq z^{*}\right\}, \\
\Omega_{2}^{*}=\bigcup_{z^{*} \in \Omega_{2}}\left\{z \in R_{+}^{n} \mid z \leq z^{*}\right\} .
\end{gathered}
$$


Then, $S=\left\{\phi \in P C \mid[\phi]_{\tau}^{+} \in \Omega_{2}^{*} \cap \Omega_{3}\right\}$ is an attracting set of (2) and $D=\left\{\phi \in P C \mid[\phi]_{\tau}^{+} \in \Omega_{1}^{*}\right\}$ is the attracting basin of $S$.

Proof. From (5) and the definitions of the above sets, then $z^{*} \in \Omega_{1}, M z^{*} \in \Omega_{2}, 0 \in \Omega_{1}^{*}, 0 \in \Omega_{2}^{*}, 0 \in \Omega_{3}$. Obviously, $\Omega_{1}, \Omega_{2}, \Omega_{1}^{*}, \Omega_{2}^{*}, \Omega_{3}$ and $\Omega_{2}^{*} \cap \Omega_{3}$ are nonempty, and so the definitions of the sets of $S$ and $D$ are valid. For any $\phi \in D$, there is a $z^{*} \in \Omega_{1}$ satisfying $[\phi]_{\tau}^{+} \leq z^{*}$. According to Theorem 4, we obtain

$$
[x(t)]^{+}=\left[x\left(t, t_{0}, \phi\right)\right]^{+} \leq M z^{*} \in \Omega_{2}, \quad \forall t \geq t_{0} .
$$

That is,

$$
\sigma \stackrel{\Delta}{=} \limsup _{t \rightarrow \infty}[x(t)]^{+} \in \Omega_{2}^{*}
$$

Then, for any given $\varepsilon>0$, there is a $T_{1}>t_{0}$ such that

$$
[x(t)]^{+} \leq \varepsilon E+\sigma, \quad t \geq T_{1} .
$$

In light of $W=\operatorname{diag}\left\{w_{i}\right\}>0$, for the above $\varepsilon>0$ and $T_{1}$, we can find an enough large $T_{2}>0$ such that

$$
\begin{gathered}
\int_{T_{2}}^{\infty} e^{-W s} d s \leq \varepsilon I, \\
\sum_{t_{0}<t_{k} \leq T_{1}} e^{-W\left(t-t_{k}\right)} \leq \varepsilon I, \quad t>T_{2} .
\end{gathered}
$$

Using (12), (13), (22), (24), and (25), we have for $t \geq \tau+T_{1}+T_{2}$,

$$
\begin{aligned}
{[x(t)]^{+} \leq } & e^{-W\left(t-t_{0}\right)} M[\phi]_{\tau}^{+}+\int_{t_{0}}^{t} e^{-W(t-s)} M p\left(\left[x_{s}\right]_{\tau}^{+}\right) d s \\
& +\sum_{t_{0}<t_{k} \leq t} M e^{-W\left(t-t_{k}\right)} q\left(\left[x\left(t_{k}^{-}\right)\right]^{+}\right) \\
\leq & e^{-W\left(t-t_{0}\right)} M[\phi]_{\tau}^{+} \\
& +\left\{\int_{t_{0}}^{t-T_{2}}+\int_{t-T_{2}}^{t}\right\} e^{-W(t-s)} M p\left(\left[x_{s}\right]_{\tau}^{+}\right) d s \\
& +\left\{\sum_{t_{0}<t_{k} \leq T_{1}}+\sum_{T_{1}<t_{k} \leq t}\right\} M e^{-W\left(t-t_{k}\right)} q\left(\left[x\left(t_{k}^{-}\right)\right]^{+}\right) \\
\leq & e^{-W\left(t-t_{0}\right)} M z^{*}+\int_{T_{2}}^{\infty} e^{-W s} M p\left(M z^{*}\right) d s
\end{aligned}
$$

$$
\begin{aligned}
& +\int_{t-T_{2}}^{t} e^{-W(t-s)} M p(\varepsilon E+\sigma) d s \\
& +M \sum_{t_{0}<t_{k} \leq T_{1}} e^{-W\left(t-t_{k}\right)} q\left(M z^{*}\right) \\
& +M \sum_{T_{1}<t_{k} \leq t} e^{-W\left(t-t_{k}\right)} q(\varepsilon E+\sigma) \\
& \leq e^{-W\left(t-t_{0}\right)} M z^{*}+\varepsilon M p\left(M z^{*}\right) \\
& +\left(I-e^{-W T_{2}}\right) W{ }^{-1} M p(\varepsilon E+\sigma)+\varepsilon M q\left(M z^{*}\right) \\
& +M\left(I-e^{-W\left(t-T_{1}\right)}\right)\left[I-e^{-W \theta}\right]^{-1} q(\varepsilon E+\sigma) \\
& \leq e^{-W\left(t-t_{0}\right)} M z^{*}+\varepsilon M\left[p\left(M z^{*}\right)+q\left(M z^{*}\right)\right] \\
& +W{ }^{-1} M p(\varepsilon E+\sigma)+M\left[I-e^{-W \theta}\right]^{-1} q(\varepsilon E+\sigma) .
\end{aligned}
$$

This implies that

$$
\begin{aligned}
& \sigma=\limsup _{t \rightarrow+\infty}[x(t)]^{+} \leq \varepsilon M\left[p\left(M z^{*}\right)+q\left(M z^{*}\right)\right] \\
&+W^{-1} M p(\varepsilon E+\sigma)+M\left[I-e^{-W \theta}\right]^{-1} q(\varepsilon E+\sigma) .
\end{aligned}
$$

Letting $\epsilon \rightarrow 0^{+}$, then

$$
\sigma \leq W^{-1} M p(\sigma)+M\left[I-e^{-W \theta}\right]^{-1} q(\sigma) .
$$

That is, $\Delta(\sigma) \geq 0$ and $\sigma \in \Omega_{3}$. Thus,

$$
\sigma \in \Omega_{2}^{*} \cap \Omega_{3} .
$$

From the definition of $\sigma$ and $S$, dist $\left(x_{t}\left(t_{0}, \phi\right), S\right) \rightarrow 0$ as $t \rightarrow$ $+\infty$. The proof is complete.

From the above theorems, we can obtain sufficient conditions ensuring global attractivity and stability in the following corollaries.

Corollary 6. Assume that $\left(H_{1}\right)-\left(H_{3}\right)$ hold with

$$
\begin{gathered}
P\left([\varphi]_{\tau}^{+}\right)=P[\varphi]_{\tau}^{+}+\mu, \\
P=\left(p_{i j}\right)_{n \times n} \geq 0, \quad \mu=\left(\mu_{1}, \ldots, \mu_{n}\right)^{T} \geq 0, \\
q\left([x]^{+}\right)=Q[x]^{+}+\nu, \\
Q=\left(q_{i j}\right)_{n \times n} \geq 0, \quad v=\left(\nu_{1}, \ldots, \nu_{n}\right)^{T} \geq 0 .
\end{gathered}
$$

If the spectral radius

$$
\rho(\Lambda)<1, \quad \text { where } \Lambda=W^{-1} M P+M\left[I-e^{-\theta W}\right]^{-1} Q,
$$

then $D=\left\{\phi \in P C \mid[\phi]_{\tau}^{+} \leq Z \triangleq(I-\Lambda)^{-1} W^{-1}(\mu+W[I-\right.$ $\left.\left.\left.e^{-\theta W}\right]^{-1} \nu\right)\right\}$ is a positive quasi-invariant set of (2), and $S=\{\phi \in$ $\left.P C \mid[\phi]_{\tau}^{+} \leq(I-\Lambda)^{-1} W^{-1} M\left(\mu+W\left[I-e^{-\theta W}\right]^{-1} \nu\right)\right\}$ is a global attracting set of (2). 
Proof. Since $p(z)=P z+\mu$ and $q(z)=Q z+\nu$, we directly calculate

$$
\begin{gathered}
\Delta(z)=M^{-1} W(\Lambda-I) z+\mu+W\left[I-e^{-\theta W}\right]^{-1} \nu \\
\Delta(M z)=W(\Lambda-I) z+\mu+W\left[I-e^{-\theta W}\right]^{-1} \nu .
\end{gathered}
$$

Without loss of generality, we assume that $\mu, v>0$. Since $\rho(\Lambda)<1,(I-\Lambda)^{-1}$ exists and $(I-\Lambda)^{-1} \geq 0$ (see [23]), and so $Z>0$. For any $\kappa>0$, we take $z^{*}=(1+\kappa) Z>0$ in Theorem 4 and verify the condition (5):

$$
\Delta\left(M z^{*}\right)=-\kappa\left(\mu+W\left[I-e^{-\theta W}\right]^{-1} \nu\right)<0 .
$$

According to Theorem 4, when $\kappa \rightarrow 0$, we deduce that $D$ is a positive quasi-invariant set of (2). Furthermore, by (33),

$$
(1+\kappa) Z \in \Omega_{1}, \quad(1+\kappa) M Z \in \Omega_{2} .
$$

From the arbitrariness of $\kappa$, we obtain $\Omega_{1}^{*}=\Omega_{2}^{*}=R_{+}^{n}$. Moreover,

$$
\begin{aligned}
\Omega_{3}= & \left\{z \in R_{+}^{n} \mid \Delta(z) \geq 0\right\} \\
= & \left\{z \in R_{+}^{n} \mid M^{-1} W(\Lambda-I) z+\mu+W\left[I-e^{-\theta W}\right]^{-1} \nu \geq 0\right\} \\
= & \left\{z \in R_{+}^{n} \mid(I-\Lambda) z \leq W^{-1} M\left(\mu+W\left[I-e^{-\theta W}\right]^{-1} \nu\right)\right\} \\
\subset & \left\{z \in R_{+}^{n} \mid z \leq(I-\Lambda)^{-1} W^{-1} M\right. \\
& \left.\quad \times\left(\mu+W\left[I-e^{-\theta W}\right]^{-1} \nu\right)\right\} .
\end{aligned}
$$

It follows from Theorem 5 that $S^{\prime}=\left\{\phi \in \mathrm{PC} \mid[\phi]_{\tau}^{+} \in \Omega_{3}\right\}$ is a global attracting set of (2) and $S$ is also a global attracting set due to $S^{\prime} \subset S$. The proof is complete.

Corollary 7. Assume that all conditions in Corollary 6 hold with $\mu=\nu=0$. Then, the zero solution $x(t)=0$ of (2) is globally asymptotically stable.

\section{Illustrative Examples}

The following illustrative examples will demonstrate the effectiveness of our results and also show the different asymptotical behaviors between the impulsive system and the corresponding continuous system.

Example 8. Consider a scalar nonlinear impulsive delay system

$$
\begin{array}{r}
\dot{x}(t)=0.2 x(t)+0.2 x^{2}(t-1)+0.1, \\
t \neq t_{k}, k \in N, t \geq t_{0}=0, \\
\Delta x=-0.6 x\left(t_{k}^{-}\right)+0.1 x^{2}\left(t_{k}^{-}\right)+0.1 \sin \left(e^{t_{k}}\right), \\
t_{k}=t_{k-1}+0.15 .
\end{array}
$$

According to Theorems 4 and 5, we have $\theta=\varrho=0.15, A=$ $0.2, B=-0.6, M=2.5, W \doteq 5.9086, p(z)=0.2 z^{2}+0.1$, $q(z)=0.1 z^{2}+0.1, \Delta(z) \doteq 1.2052 z^{2}-2.3634 z+1.1052$, and so

$$
\begin{gathered}
\Omega_{1}=\left\{z \in R_{+} \mid \Delta(M z)<0\right\}=(0.3079,0.4765), \\
\Omega_{1}^{*}=[0,0.4765], \\
\Omega_{2}=\left\{z \in R_{+} \mid \Delta(z)<0\right\}=(0.7698,1.1913), \\
\Omega_{2}^{*}=[0,1.1913] \\
\Omega_{3}=\left\{z \in R_{+} \mid \Delta(z) \geq 0\right\}=[0,0.7698] \cup[1.1913,+\infty), \\
\Omega_{2}^{*} \cap \Omega_{3}=[0,0.7698]
\end{gathered}
$$

Thus, $S=\left\{\phi \in \mathrm{PC} \mid[\phi]_{\tau}^{+} \leq 0.7698\right\}$ is an attracting set of (36), and $D=\left\{\phi \in \mathrm{PC} \mid[\phi]_{\tau}^{+} \leq 0.4765\right\}$ is an attracting basin of $S$. However, solutions of the corresponding continuous system (i.e., $\Delta x=0$ in (36)) may be unbounded. Taking the initial condition $\phi(s)=0.2, s \in[-1,0]$, Figure 1 shows the different asymptotic behavior between the solution of (36) with no impulse and one with impulses.

Example 9. Consider a 2-dimensional impulsive delay system

$$
\begin{array}{r}
\dot{x}_{1}(t)=x_{1}(t)+0.5 \sin \left(x_{1}(t-1)\right)-0.4 x_{2}(t-1)-0.5, \\
t \geq 0, \\
\dot{x}_{2}(t)=-4 x_{2}(t)-0.5 x_{1}(t-1)+0.4 \cos \left(x_{2}(t-1)\right)+0.5, \\
t \neq t_{k}, \\
\Delta x_{1}=-0.5 x_{1}\left(t_{k}^{-}\right)+0.1 \cos \left(x_{1}\left(t_{k}^{-}\right)\right)+0.5 \sin \left(e^{t_{k}}\right), \\
t_{k}=0.1 k, \\
\Delta x_{2}=0.1 x_{2}\left(t_{k}^{-}\right)+0.2 \sin \left(x_{2}\left(t_{k}^{-}\right)\right)-0.5 \cos \left(e^{t_{k}}\right), \\
k \in N .
\end{array}
$$

According to Corollary 6, we have $\theta=\varrho=0.1$, $A=\operatorname{diag}\{1,-4\}, \quad B=\operatorname{diag}\{-0.5,0.1\}, \quad M=\operatorname{diag}\{2,1\}$, $W=\operatorname{diag}\{5.9315,3.0469\}, p(z)=P z+\mu, q(z)=Q z+\nu$, $\Lambda=W^{-1} M P+M\left[I-e^{-\theta W}\right]^{-1} Q$, where

$$
\begin{gathered}
P=\left(\begin{array}{ll}
0.5 & 0.4 \\
0.5 & 0.4
\end{array}\right), \quad Q=\left(\begin{array}{cc}
0.1 & 0 \\
0 & 0.2
\end{array}\right), \\
\mu=\nu=\left(\begin{array}{l}
0.5 \\
0.5
\end{array}\right), \quad \Lambda=\left(\begin{array}{ll}
0.6156 & 0.1349 \\
0.1641 & 0.8928
\end{array}\right),
\end{gathered}
$$

and so $\rho(\Lambda)=0.9575<1$. Therefore, $D=\{\phi \in \mathrm{PC} \mid$ $\left.[\phi]_{\tau}^{+} \leq(1.7105,2.5228)^{T}\right\}$ is a positive quasi-invariant set of (38), and $S=\left\{\phi \in \mathrm{PC} \mid[\phi]_{\tau}^{+} \leq(1.8637,2.8720)^{T}\right\}$ is a global attracting set of (38). Figure 2 shows the asymptotic properties of solutions of (38) under the different initial conditions. 


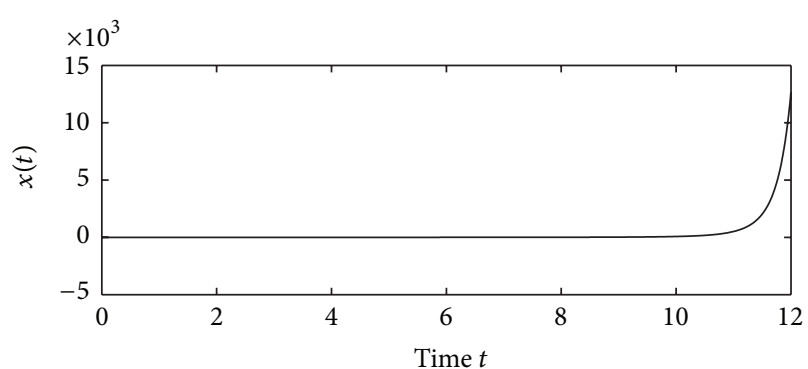

(a)

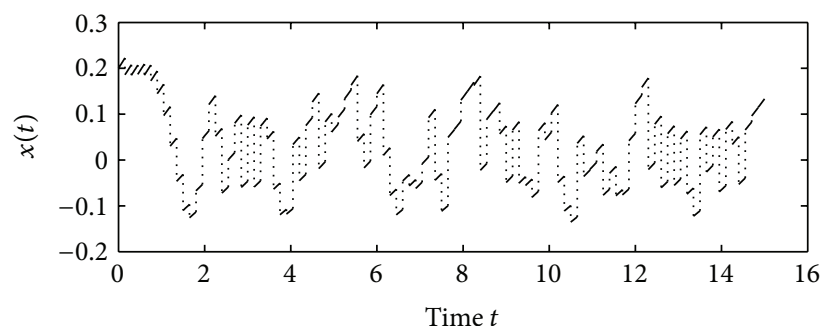

(b)

FIGURE 1: The trajectory of (36) with: (a) no impulse (i.e., $\Delta x=0$ ) and (b) impulses.

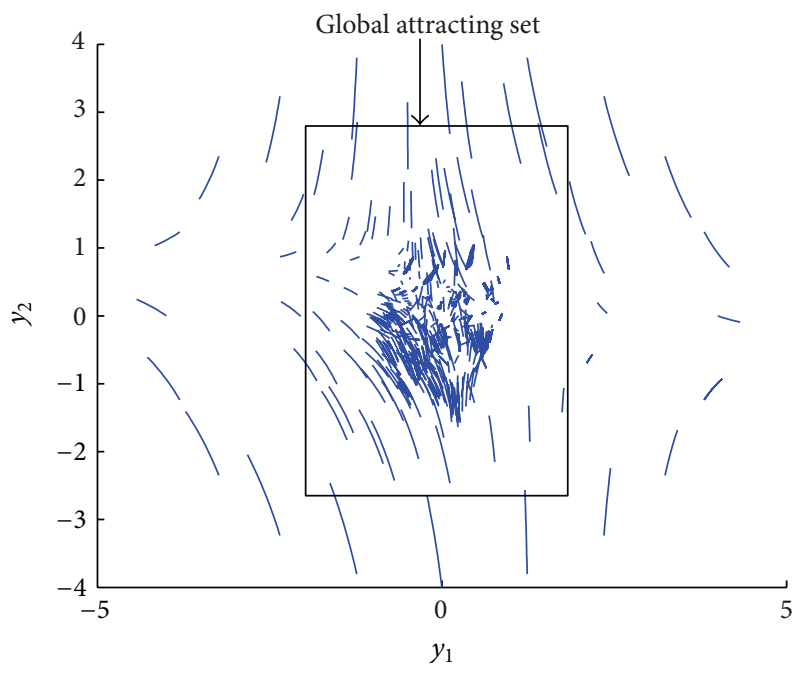

FIGURE 2: Global attracting set of (38).

\section{Acknowledgments}

This work is supported partially by the National Natural Science Foundation of China under Grant nos. 10971240, 61263020, and 61004042, the Key Project of Chinese Education Ministry under Grant no. 212138, the Natural Science Foundation of Chongqing under Grant CQ CSTC 2011BB0117, and the Foundation of Science and Technology project of Chongqing Education Commission under Grant KJ120630.

\section{References}

[1] A. M. Somoilenko and N. A. Perslyuk, Differential Equations With Impulse Effect, Visca Skala, Kiev, Ukraine, 1987.

[2] V. Lakshmikantham, D. D. Baĭnov, and P. S. Simeonov, Theory of Impulsive Differential Equations, vol. 6, World Scientific, Singapore, 1989.

[3] D. D. Baĭnov and P. S. Simeonov, Systems with Impulse Effect: Stability Theory and Applications, Ellis Horwood, Chichester, UK, 1989.
[4] K. Gopalsamy and B. G. Zhang, "On delay differential equations with impulses," Journal of Mathematical Analysis and Applications, vol. 139, no. 1, pp. 110-122, 1989.

[5] A. Anokhin, L. Berezansky, and E. Braverman, "Exponential stability of linear delay impulsive differential equations," Journal of Mathematical Analysis and Applications, vol. 193, no. 3, pp. 923-941, 1995.

[6] A. Zhao and J. Yan, "Asymptotic behavior of solutions of impulsive delay differential equations," Journal of Mathematical Analysis and Applications, vol. 201, no. 3, pp. 943-954, 1996.

[7] J. H. Shen, "Razumikhin techniques in impulsive functionaldifferential equations," Nonlinear Analysis, vol. 36, no. 1, pp. 119$130,1999$.

[8] Z. H. Guan and G. Chen, "On delayed impulsive Hopfield neural networks," Neural Networks, vol. 12, pp. 273-280, 1999.

[9] X. Liu and G. Ballinger, "Uniform asymptotic stability of impulsive delay differential equations," Computers and Mathematics with Applications, vol. 41, no. 7-8, pp. 903-915, 2001.

[10] J. S. Yu, "Stability for nonlinear delay differential equations of unstable type under impulsive perturbations," Applied Mathematics Letters, vol. 14, no. 7, pp. 849-857, 2001.

[11] Z. Luo and J. Shen, "Impulsive stabilization of functional differential equations with infinite delays," Applied Mathematics Letters, vol. 16, no. 5, pp. 695-701, 2003.

[12] J. Yan, A. Zhao, and J. J. Nieto, "Existence and global attractivity of positive periodic solution of periodic single-species impulsive Lotka-Volterra systems," Mathematical and Computer Modelling, vol. 40, no. 5-6, pp. 509-518, 2004.

[13] Z. Yang and D. Xu, "Existence and exponential stability of periodic solution for impulsive delay differential equations and applications," Nonlinear Analysis, vol. 64, no. 1, pp. 130-145, 2006.

[14] Z. Yang and D. Xu, "Stability analysis and design of impulsive control systems with time delay," IEEE Transactions on Automatic Control, vol. 52, no. 8, pp. 1448-1454, 2007.

[15] Z. Yang and D. Xu, "Robust stability of uncertain impulsive control systems with time-varying delay," Computers and Mathematics with Applications, vol. 53, no. 5, pp. 760-769, 2007.

[16] Z. Yang, T. Huang, L. Zhang, and Z. Yang, "On networked control of impulsive hybrid systems," Computers and Mathematics with Applications, vol. 61, no. 8, pp. 2076-2080, 2011.

[17] Y. Zhang and J. Sun, "Stability of impulsive functional differential equations," Nonlinear Analysis, vol. 68, no. 12, pp. 3665$3678,2008$. 
[18] J. Zhou and Q. Wu, "Exponential stability of impulsive delayed linear differential equations," IEEE Transactions on Circuits and Systems II, vol. 56, no. 9, pp. 744-748, 2009.

[19] D. Xu and Z. Yang, "Attracting and invariant sets for a class of impulsive functional differential equations," Journal of Mathematical Analysis and Applications, vol. 329, no. 2, pp. 1036-1044, 2007.

[20] Z. Ma and X. Wang, "A new singular impulsive delay differential inequality and its application," Journal of Inequalities and Applications, vol. 2009, Article ID 461757, 13 pages, 2009.

[21] B. Li, "Invariant and attracting sets of non-autonomous impulsive neutral integro-differential equations," Electronic Journal of Qualitative Theory of Differential Equations, vol. 67, pp. 1-18, 2012.

[22] L. $\mathrm{Xu}$ and $\mathrm{D} . \mathrm{Xu}$, " $P$-attracting and $p$-invariant sets for a class of impulsive stochastic functional differential equations," Computers and Mathematics with Applications, vol. 57, no. 1, pp. 54-61, 2009.

[23] R. A. Horn and C. R. Johnson, Matrix Analysis, Cambridge University Press, 1985. 


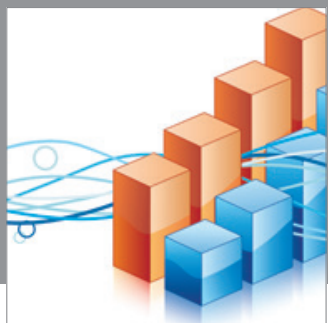

Advances in

Operations Research

mansans

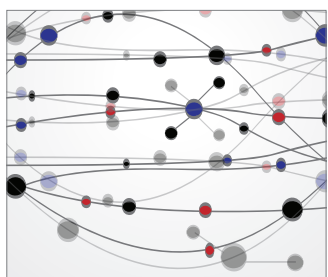

The Scientific World Journal
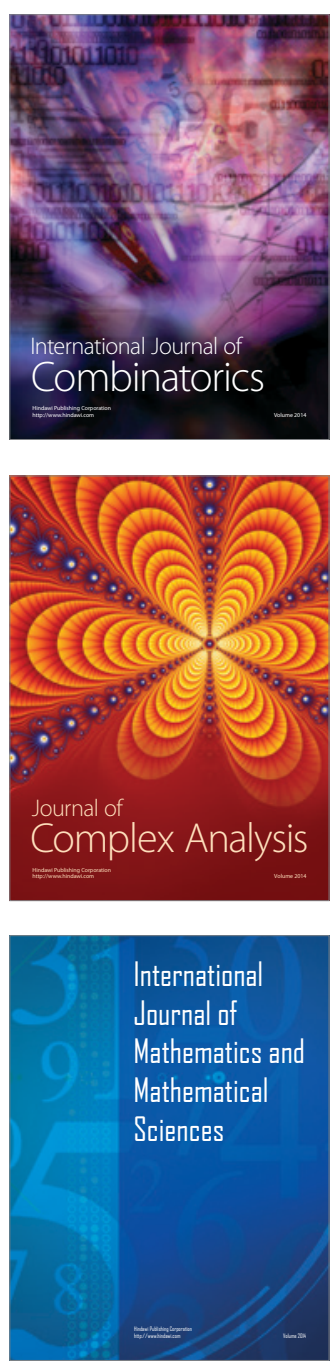
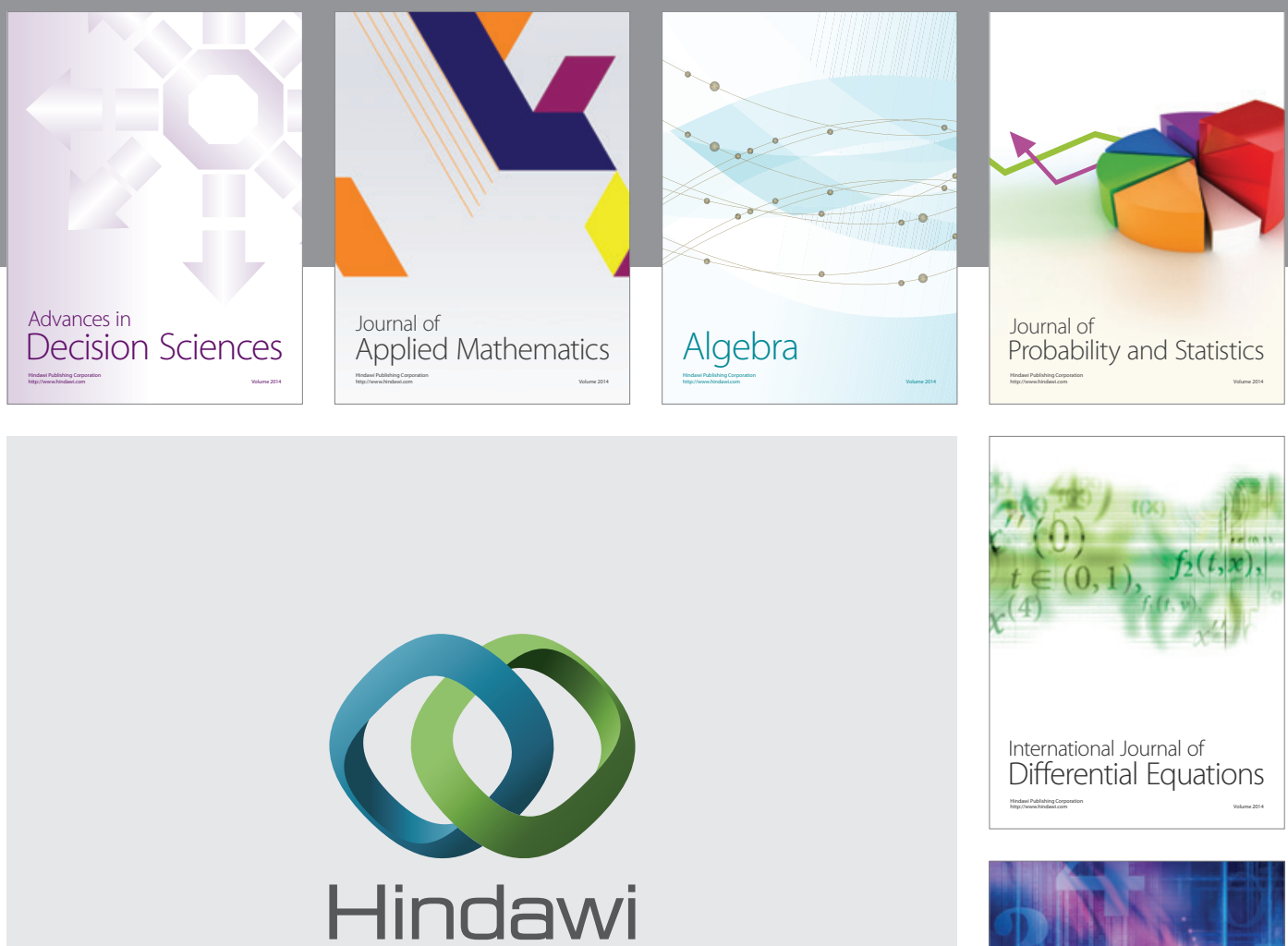

Submit your manuscripts at http://www.hindawi.com
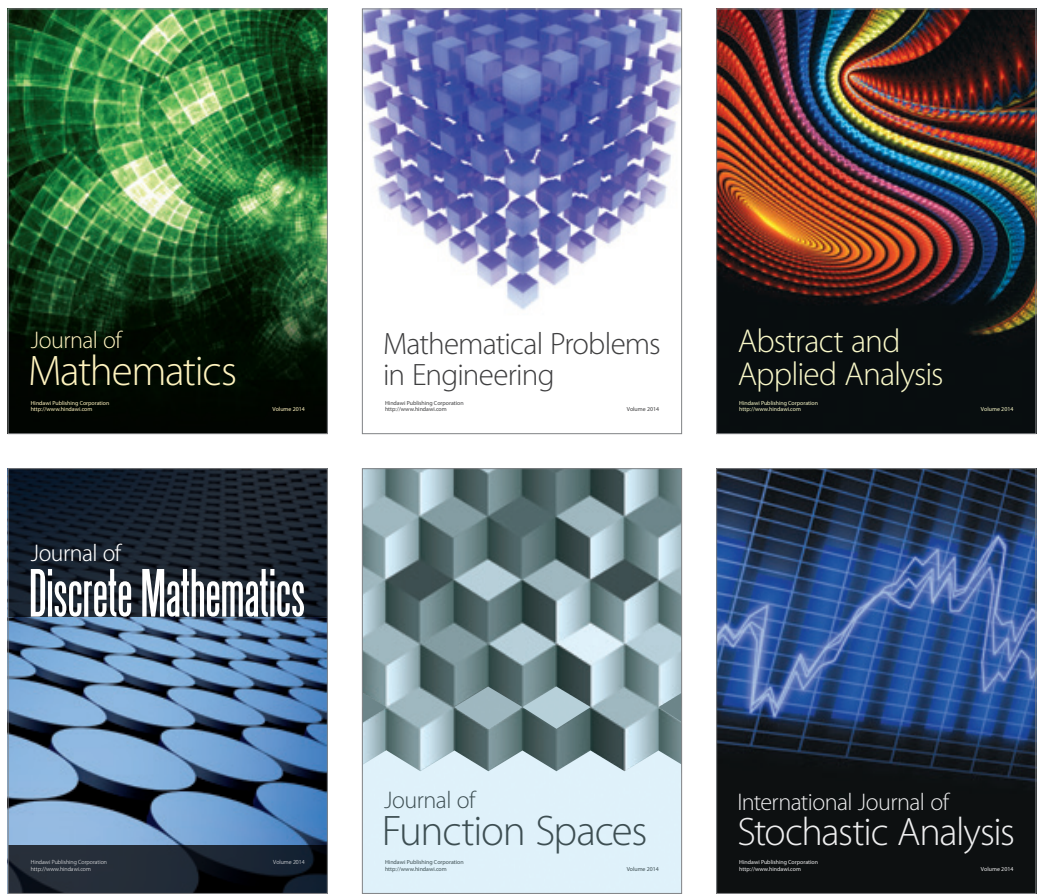

Journal of

Function Spaces

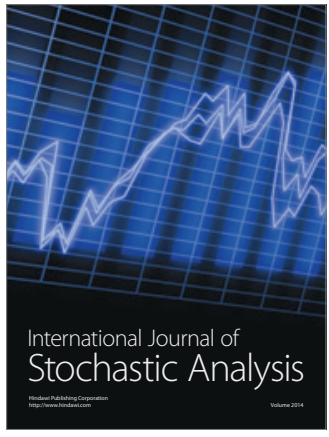

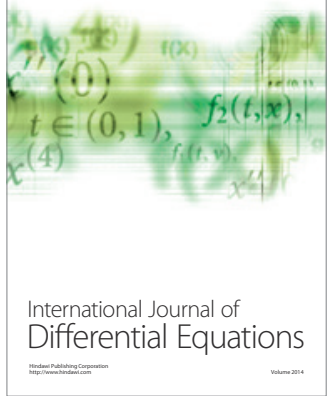
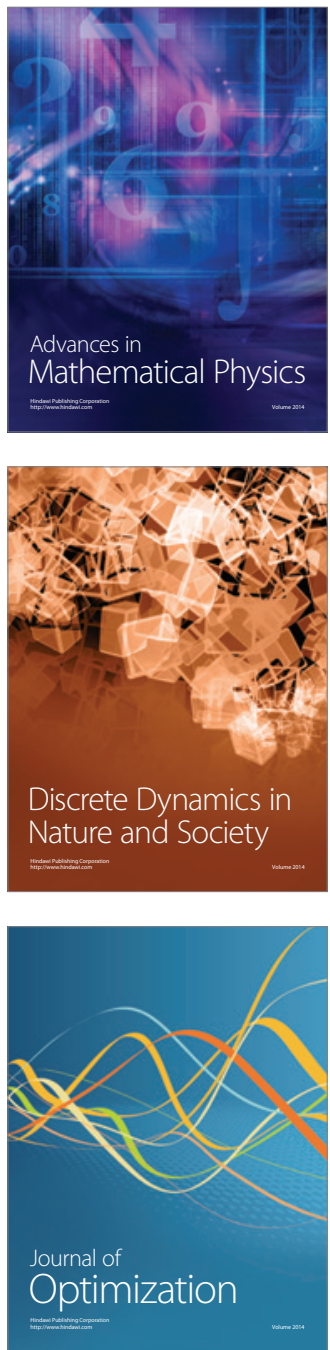